\title{
Solution Structure of Oxidized Horse Heart Cytochrome $\mathrm{c}^{\dagger, \perp}$
}

\author{
Lucia Banci,, Ivano Bertini, ${ }^{*}$, Harry B. Gray, ${ }^{\S}$ Claudio Luchinat," Tim Reddig, ${ }^{\ddagger}$ Antonio Rosato,, and \\ Paola Turano \\ Department of Chemistry, University of Florence, Via Gino Capponi 7, 50121, Florence, Italy, Beckmann Institute, \\ California Institute of Technology, Pasadena, California 91125, and Department of Soil Science and Plant Nutrition, \\ University of Florence, Florence, Italy
}

Received March 27, 1997; Revised Manuscript Received May 27, $1997^{\circledR}$

\begin{abstract}
The solution structure of oxidized horse heart cytochrome c was obtained at $\mathrm{pH} 7.0$ in 100 $\mathrm{mM}$ phosphate buffer from 2278 NOEs and 241 pseudocontact shift constraints. The final structure was refined through restrained energy minimization. A 35-member family, with RMSD values with respect to the average structure of $0.70 \pm 0.11 \AA$ and $1.21 \pm 0.14 \AA$ for the backbone and all heavy atoms, respectively, and with an average penalty function of $130 \pm 4.0 \mathrm{~kJ} / \mathrm{mol}$ and $84 \pm 3.7 \mathrm{~kJ} / \mathrm{mol}$ for NOE and pseudocontact shift constraints, respectively (corresponding to a target function of $0.9 \AA^{2}$ and 0.2 $\AA^{2}$ ), was obtained. The solution structure is somewhat different from that recently reported (Qi et al., 1996) and appears to be similar to the X-ray structure of the same oxidation state (Bushnell et al., 1990). A noticeable difference is a rotation of $17 \pm 8^{\circ}$ of the imidazole plane between solid and solution structure. Detailed and accurate structural determinations are important within the frame of the current debate of the structural rearrangements occurring upon oxidation or reduction. From the obtained magnetic susceptibility tensor a separation of the hyperfine shifts into their contact and pseudocontact contributions is derived and compared to that of the analogous isoenzyme from $S$. cerevisiae and to previous results.
\end{abstract}

We have been recently involved in the solution structure determination of oxidized and reduced Saccharomyces cerevisiae iso-1-cytochrome c (Banci et al., 1997a; Baistrocchi et al., 1996) and of the cyanide adduct of its Met80Ala mutant in the oxidized form (Banci et al., 1995a). The latter solution structure showed that the global folding of the protein upon substitution of the axial methionine ligand with an alanine is substantially unaffected and that the distal side interactions stabilize small molecules bound to the iron ion. The principal aim of the work on the wild type $S$. cerevisiae cytochrome $\mathrm{c}$ was to obtain information on the structural differences between the oxidized and the reduced forms, which are possibly related to the ability of these molecules to act as electron transfer agents.

There has been a large debate in the literature (Scott \& Mauk, 1996) on the nature and extent of the differences between oxidized and reduced cytochrome c. Crystal structure data and our solution structures point out to a few minor, but probably significant, differences in some tertiary structure elements. In particular, we have found that the two forms of $S$. cerevisiae cytochrome c in solution (Banci et al., 1997a; Baistrocchi et al., 1996) differ only because of the H-bond network involving propionate-7 and for the orientation of a few side chains which have been proposed to be involved in the interaction with biological redox partners (Pelletier \& Kraut, 1992). All the elements of

This work was supported by the EC BIOMED program (contract CT BMH4-CT96-1492) and by Italian CNR (Tecnologie Chimiche Innovative; contract 96.00802CT03).

${ }^{\perp}$ The coordinates of the average energy-minimized PSEUDOREM structure have been deposited in the Brookhaven Protein Data Bank (filename 1AKK).

$\ddagger$ Department of Chemistry, University of Florence.

$\S$ California Institute of Technology.

" Department of Soil Science, University of Florence.

${ }^{\otimes}$ Abstract published in Advance ACS Abstracts, July 15, 1997. secondary structure are maintained, in accordance with the data on the X-ray structures of $S$. cerevisiae (Berghuis \& Brayer, 1992; Langen et al., 1992) and tuna (Takano \& Dickerson, 1981b; Takano \& Dickerson, 1981a) cytochromes c. Recently, the solution structure of oxidized horse heart cytochrome c (hh cyt c, hereafter) has been solved (Qi et al., 1996). It seems substantially different, even for what concerns the secondary structure, from the available solution structure of the reduced form (Qi et al., 1994) and from the $\mathrm{X}$-ray crystal structure of the oxidized protein (Bushnell et al., 1990). We felt that some of the differences could be ascribed to the poor resolution of the solution structure of the oxidized species due to the lack of few strategic experimental constraints. In order to obtain a deeper insight into the problem of the structural reorganization associated with electron transfer, which may be obtained through the comparison of the solution structures of several related electron transfer proteins, we have solved again the structure of oxidized hh cyt $\mathrm{c}$. We have also used the pseudocontact shifts as structural constraints that have provided accurate information on the magnetic susceptibility tensor. From these data, the separation of the hyperfine shifts into their contact and pseudocontact contributions is obtained.

\section{EXPERIMENTAL SECTION}

NMR Sample Preparation. Horse heart cytochrome c (Type VI) was obtained from Sigma Chemical Co. and used without further purification. The ${ }^{1} \mathrm{H}$ NMR samples were prepared by dissolving the lyophilized protein in $100 \mathrm{mM}$ phosphate buffer at $\mathrm{pH} 7.0$ to give $10-15 \mathrm{mM}$ solutions. The $\mathrm{pH}$ of protein samples prepared for NMR spectroscopy was adjusted by addition of small volumes of concentrated solutions of $\mathrm{NaOH}$ and $\mathrm{H}_{3} \mathrm{PO}_{4}$. The $\mathrm{pH}$ was measured with an Orion Model $720 \mathrm{pH}$ meter and a Microelectrodes, Inc., Model MI-410 microcombination $\mathrm{pH}$ probe. 
NMR Spectroscopy. The ${ }^{1} \mathrm{H}$ NMR spectra were recorded on a Bruker DRX 500 and AMX 600 spectrometers operating at 500.13 and $600.13 \mathrm{MHz}$, respectively. The $500 \mathrm{MHz}{ }^{1} \mathrm{H}$ NMR 1D spectra were recorded using the superWEFT (water-eliminated Fourier transform) (Inubushi \& Becker, 1983) pulse sequence with recycle delay ranging from 200 to $300 \mathrm{~ms}$. The ${ }^{1} \mathrm{H}$ nuclear Overhauser effect (NOE) experiments (at $500 \mathrm{MHz}$ ) were performed with the superWEFT pulse sequence and were collected using standard methodology (Banci et al., 1989).

$600 \mathrm{MHz}{ }^{1} \mathrm{H}$ NMR spectra were obtained by using presaturation during relaxation delay $(0.8-1.2 \mathrm{~s})$. TPPI NOESY (Macura et al., 1982; Marion \& Wüthrich, 1983) maps at $293 \mathrm{~K}$ in $\mathrm{D}_{2} \mathrm{O}$ solution were recorded on a spectral width of $40 \mathrm{ppm}$ with a recycle time of $600 \mathrm{~ms}$ and a mixing time of $100 \mathrm{~ms}$. To optimize the observation of connectivities in the diamagnetic region, NOESY maps in $\mathrm{H}_{2} \mathrm{O}$ solutions were recorded on a smaller spectral width (18 ppm), with recycle times of $1.2 \mathrm{~s}$ and mixing times of $100 \mathrm{~ms}$. Analogously, TPPI cleanTOCSY (Bax \& Davis, 1985) experiments, with presaturation during the relaxation delay, were recorded over the spectral width of $18 \mathrm{ppm}$ (recycle time of $1.2 \mathrm{~s}$ and spin lock times of $45 \mathrm{~ms}$ ).

All 2D spectra consisted of $4 \mathrm{~K}$ data points in the $\mathrm{F} 2$ dimension. From 800 to 1024 experiments were recorded in the F1 dimension, using 64-192 scans per experiment. Raw data were multiplied in both dimensions by a pure cosine-squared and Fourier-transformed to obtain $2048 \times$ 2048 real data points. A polynomial base-line correction was applied in both directions.

Data processing was performed using a standard Bruker software package. The 2D maps were analyzed on IBM RISC 6000 computers with the aid of the program XEASY (ETH, Zürich) (Eccles et al., 1991).

Proton-Proton Distance Constraints. The volumes of the NOESY cross peaks between assigned resonances were obtained by manual integration, with the elliptical integration routine implemented in the program XEASY. Most of the dipolar connectivities were taken from the $100 \mathrm{~ms}$ NOESY map in $\mathrm{H}_{2} \mathrm{O}$ solution at $293 \mathrm{~K}$. Connectivities whose volumes could be better measured in the spectrum in $\mathrm{D}_{2} \mathrm{O}$ solution were scaled referring to a few cross peaks whose volumes could be accurately measured in both spectra.

NOESY cross peak intensities were converted into upper limits of interatomic distances by following the methodology of the program CALIBA (Güntert et al., 1991). 1D NOEs involving paramagnetic signals (for a total of 28 NOEs) were converted into upper distance limits of 3.5, 5.0, or $6.5 \AA$ for proton pairs connected by strong, medium, or weak NOEs, respectively.

Pseudocontact-Shift Constraints. The hyperfine shift, i.e., the difference in shift of a proton in the paramagnetic system from that in an analogous diamagnetic system, includes contact and pseudocontact contributions (La Mar et al., 1973; Bertini \& Luchinat, 1986; Bertini \& Turano, 1994; Bertini \& Luchinat, 1996). The contact coupling is due to the presence of unpaired spin density on the resonating nucleus and vanishes a few chemical bonds away from the metal. The pseudocontact contribution $\left(\delta_{\mathrm{pc}}\right)$ arises from the magnetic susceptibility anisotropy and depends on the nuclear position with respect to the principal axes of the magnetic susceptibility tensor. Within the metal-centered point-dipole pointdipole approximation, the following equation holds:

$$
\delta_{\mathrm{pc}}=\frac{1}{12 \pi r_{\mathrm{i}}^{3}}\left[\Delta \chi_{\mathrm{ax}}\left(3 n_{\mathrm{i}}^{2}-1\right)+\frac{3}{2} \Delta \chi_{\mathrm{rh}}\left(l_{\mathrm{i}}^{2}-m_{\mathrm{i}}^{2}\right)\right]
$$

where $\Delta \chi_{\mathrm{ax}}$ and $\Delta \chi_{\mathrm{rh}}$ are the axial and the rhombic anisotropy of the magnetic susceptibility induced by the paramagnetic ion, $r_{\mathrm{i}}$ is the distance of the nucleus $\mathrm{i}$ from the metal ion, and $l_{\mathrm{i}}, m_{\mathrm{i}}$, and $n_{\mathrm{i}}$ are the direction cosines of the position vector of atom $\mathrm{i}\left(\mathbf{r}_{\mathrm{i}}\right)$ with respect to the orthogonal reference system formed by the principal axes of the magnetic susceptibility tensor.

Pseudocontact shifts were determined by subtracting the chemical shifts measured in the reduced species (Wand et al., 1989) from those of the oxidized one. The resonances of cysteines 14 and 17, of histidine 18, and of methionine 80 would experience non-negligible contact shifts and therefore were not included in calculations.

Until now, if a single structure was available, five independent parameters (i.e., $\Delta \chi_{\mathrm{ax}}, \Delta \chi_{\mathrm{rh}}$, and three independent direction cosines that define the principal directions of the $\chi$ tensor with respect to a metal-centered axis system) were determined by finding the best fit of eq 1 to a set of pseudocontact shifts (Williams et al., 1985; Feng et al., 1990; Gao et al., 1991; Emerson \& La Mar, 1990; Rajarathnam et al., 1992; La Mar et al., 1995; Banci et al., 1995b; Gochin \& Roder, 1995). For a family of $\mathrm{N}$ structures, an average magnetic susceptibility tensor can be determined by fitting an extended set of eqs 1 with the $N \mathbf{r}_{\mathrm{i}}$ vectors obtained for each structure of the family to the same set of experimental pseudocontact shift values (Banci et al., 1996). In a continuous development of the use of pseudocontact shifts for structure determination, we propose to fit eq 1 versus the numerous experimental data with eight independent parameters, namely the above five, plus the coordinates of the origin of the magnetic anisotropy tensor itself (which, when a single structure is used in the fitting, coincide with the coordinates of the metal ion). In this way the position of the metal ion is defined by experimental constraints only. This calculation was performed through the program FANTASIA (Banci et al., 1996; Banci et al., 1997c). In order to evaluate the discrepancies between calculated and experimental pseudocontact shifts, a target function (TF) is defined following the definition of the target function relative to the NOEs of the DYANA program:

$$
\begin{aligned}
\mathrm{TF}=\sum_{i} w_{i}\left[\Theta\left(\left|\delta_{\mathrm{exp}}^{\mathrm{pc}}-\delta_{\text {calc }}^{\mathrm{pc}}\right|, \text { toll }\right)\right]^{2} \\
\Theta(x, \text { toll })=\left\{\begin{array}{l}
0 \text { if toll }>x \\
(x-\text { toll }) \text { if toll }<x
\end{array}\right.
\end{aligned}
$$

where the summation spans over all pseudocontact shift constraints, $w_{i}$ is the weight of the $i$ th constraint (all taken equal in this work), $\delta_{\exp }^{\mathrm{pc}}$ is the $i$ th measured pseudocontact shift, $\delta_{\text {calc }}^{\mathrm{pc}}$ is the corresponding calculated pseudocontact shift, toll is the tolerance given to the pseudocontact shift constraints. Calculated and experimental shifts are considered equal if the absolute value of their difference is smaller than the tolerance. The tolerance should be large enough to include possible deviations from the metal-centered approximation and possible changes in the diamagnetic shift between the oxidized and reduced form of the protein (e.g. due to minor structural changes or to the different hydrogen 
bonding network of amide protons). The choice of the value of the weight of pseudocontact shift constraints relative to that of NOE constraints is crucial for structure calculations. Moreover, it is largely dependent on the choice of the tolerance. It seems reasonable to weight the pseudocontact shift and the NOE constraints equally (i.e. $w_{i}=1$ ) and to adjust only the value of the tolerance. The latter should be set in a way such that the introduction of the pseudocontact shift constraints does not affect much the value of the target function due to the NOE constraints, and the contribution to the total target function of the shift constraints is comparable to that of the other constraints. In this work, tolerance values of $0.3 \mathrm{ppm}$ and $0.5 \mathrm{ppm}$ were imposed to nonexchangeable and exchangeable protons, respectively (the tolerance imposed to exchangeable protons is larger as their diamagnetic shift is more dependent on experimental conditions, like $\mathrm{pH}$, temperature, etc., than the shift of nonexchangeable protons).

The $\Delta \chi_{\mathrm{ax}}$ and $\Delta \chi_{\mathrm{rh}}$ tensor parameters derived from FANTASIA calculations were used as input in structure calculations. Since the program used for structure calculations (see later) is capable to adjust the origin and the orientation of the magnetic anisotropy tensor, it is useless to input the other parameters. With the old program (see later) used for the structure determination of $S$. cerevisiae cytochrome c, $\Delta \chi_{\mathrm{ax}}, \Delta \chi_{\mathrm{rh}}$ and the three direction cosines were needed as an input, and the origin of the tensor was fixed onto the iron ion (Banci et al., 1997a).

Structure Calculations. Structure calculations were performed with the program DYANA (Güntert et al., 1997), available from the web site of Kurt Wüthrich's group (http: //odin.ethz.ch/wuthrich/software/). We added to this program a module to use pseudocontact shifts as structural constraints (Güntert, P., Banci, L., Bertini, I., Luchinat, C. et al. manuscript in preparation), which will be referred to as PSEUDYANA. The results are equivalent to those obtained through the programs DIANA (Güntert \& Wüthrich, 1991) and PSEUDIANA (Banci et al., 1996), respectively. A residue containing the heme moiety, defined as previously described (Banci et al., 1995a; Baistrocchi et al., 1996; Banci et al., 1997a), was added to the standard library. Following the procedure applied to $S$. cerevisiae cytochrome c, upper $(2.10 \AA)$ and lower $(1.80 \AA)$ distance limits from the $\alpha$ carbons of thioethers 2 and 4 of the heme moiety to the $\mathrm{S} \gamma$ of cysteines 14 and 17, respectively, were used in the calculations to link the heme moiety to the bound cysteines; an upper $(2.50 \AA)$ and a lower $(2.20 \AA)$ distance limit between the $\mathrm{S} \delta$ of methionine 80 and the iron ion of the heme were introduced as well.

The procedure with which the pseudocontact shift constraints are used in structural calculations is slightly different from that previously reported for $S$. cerevisiae cytochrome c (Banci et al., 1997a) and for the cyanide adduct of its Met80Ala variant (Banci et al., 1996). In the present case the metal center and the three atoms used to define the directions of the magnetic susceptibility tensor with respect to the molecular frame are introduced as a further residue. To do this, a special residue (LTNS), containing a pseudoatom $(\mathrm{PCN})$ defining the center of the tensor and three pseudoatoms (AX, AY, AZ) placed at unitary distance from it in a way such that the three PCN-(AX, AY, AZ) directions are mutually orthogonal, was added to the standard DYANA library. The three above directions define the directions of three principal axes of the tensor. The LTNS residue was linked to the polypeptide chain through a long chain of special linker residues contained in the standard library. The linker residues and the LTNS residue are made up by pseudoatoms with a null Van der Waals radius and thus can penetrate the polypeptide chain freely. During PSEUDYANA calculations (see later), the program recalculates the position of the metal center together with the orientation of the magnetic axes, the only input data being the $\Delta \chi_{\mathrm{ax}}$ and $\Delta \chi_{\text {rh }}$ tensor parameters resulting from FANTASIA, and the pseudocontact shift values. An upper distance limit of 0.2 $\AA$ between the PCN pseudoatom and the iron ion was introduced. In the very first PSEUDYANA runs, when the number of distance constraints was not high enough to give well-defined structures, this procedure ensures the correct positioning of the tensor origin. Indeed, in the calculations performed with a more extensive set of NOE constraints, the eight-parameter fitting recalculates a position for the center of the magnetic axes that is the same as that of the iron atom of our library.

A first magnetic susceptibility tensor was obtained through an eight-parameter fit using a family of structures calculated with NOE constraints alone. The magnetic susceptibility tensor was re-evaluated after each PSEUDYANA run and used in the following calculations.

Another difference with respect to the use of pseudocontact shifts in the previous procedure (Banci et al., 1997a) is that we have now introduced the shift constraints from the beginning of structure calculations (i.e. starting from random structures, and inputting $\Delta \chi$ parameters derived from a previous family of structures), whereas previously pseudocontact shift constraints were used to refine structures already folded. This is allowed by the higher efficiency of the DYANA program with respect to DIANA.

An amount of 2278 distance constraints (Supporting Information) derived from 2D NOESY and 1D NOE connectivities and 241 pseudocontact shifts (see later; Supporting Information) were used in PSEUDYANA calculations. As already discussed, the relative weight of the two types of constraints was equal. Thirty-eight stereospecific assignments (Supporting Information) were obtained through the program GLOMSA (Güntert et al., 1991). Four hundred random structures were annealed in sixteen thousand steps using the above constraints. The thirty-five structures with the lowest target function were included in the family.

Finally, restrained energy minimization (REM) with the Sander module of Amber (Pearlman \& Case, 1991) was applied to 35 structures. Pseudocontact shifts were included as constraints by means of a modified Sander module (PSEUDOREM) (Banci et al., 1997c).

Structure calculations and analyses were performed on an IBM SP02 parallel computer.

Computer-Aided Assignment. To ease the assignment of NOESY cross peaks, the structure with lowest target function obtained from a preliminary structure calculation with a limited number of NOE's was used as an input model for NOESY back-calculation through the program CORMA (Borgias et al., 1989). This program is based on relaxation matrix calculations which allow the evaluation of NOE intensities between protons of a given structure. A single correlation time of $6 \mathrm{~ns}$ was used. A number of cross peaks, which had not been identified yet, between protons whose 


\begin{tabular}{|c|c|c|c|c|}
\hline residue & $\mathrm{HN}$ & $\mathrm{H} \alpha$ & $\mathrm{H} \beta$ & others \\
\hline Glu-4 & & & 2.009 & \\
\hline Lys-5 & & & & $0.861(\mathrm{H} \gamma), 2.844(\mathrm{H} \delta), 3.124(\mathrm{H} \epsilon)$ \\
\hline Lys-7 & & & & $1.391(\mathrm{H} \gamma), 0.868(\mathrm{H} \delta)$ \\
\hline Lys- 8 & & & & $1.301(\mathrm{H} \delta), 2.867(\mathrm{H} \epsilon)$ \\
\hline Gln-12 & & & & $6.769,7.384(\mathrm{HN})$ \\
\hline Lys-13 & & & & $2.654(\mathrm{H} \epsilon)$ \\
\hline Gln-16 & & & & $6.903,7.612(\mathrm{HN})$ \\
\hline Cys-17 & & & 2.069 & \\
\hline Glu-21 & & & 2.513 & \\
\hline Lys-22 & & & & $0.699(\mathrm{H} \gamma), 1.109(\mathrm{H} \delta), 2.905(\mathrm{H} \epsilon)$ \\
\hline Lys-25 & & & & $1.636,1.592(\mathrm{H} \gamma), 1.766(\mathrm{H} \delta), 3.071(\mathrm{H} \epsilon)$ \\
\hline Lys-27 & & & 1.469 & \\
\hline Pro-30 & & & 1.694 & $-1.088(\mathrm{H} \gamma)$ \\
\hline Leu-35 & & & 1.416 & \\
\hline Arg-38 & & & & $1.849(\mathrm{H} \gamma), 3.141(\mathrm{H} \delta)$ \\
\hline Lys-39 & & & 1.547 & $1.277(\mathrm{H} \gamma)$ \\
\hline Gln-42 & & & & $6.866,7.412(\mathrm{HN})$ \\
\hline Pro-44 & & 4.370 & $1.987,2.299$ & $2.236,2.009(\mathrm{H} \gamma), 3.815(\mathrm{H} \delta)$ \\
\hline Tyr-48 & & & & $6.932(\mathrm{H} \delta), 5.561(\mathrm{H} \epsilon)$ \\
\hline Lys-53 & & & & $1.735(\mathrm{H} \delta), 2.896(\mathrm{H} \epsilon)$ \\
\hline Gln-54 & & & & $6.985,7.434(\mathrm{HN})$ \\
\hline Lys-55 & & & 1.834 & $1.633(\mathrm{H} \gamma), 1.761(\mathrm{H} \delta), 3.064(\mathrm{H} \epsilon)$ \\
\hline Thr-58 & & & & $8.159(\mathrm{OH})$ \\
\hline Lys-60 & & & & $1.370(\mathrm{H} \gamma), 1.545(\mathrm{H} \delta), 2.291(\mathrm{H} \epsilon)$ \\
\hline Glu-61 & & & & $2.444(\mathrm{H} \gamma)$ \\
\hline Glu-62 & & & 1.953 & $2.287,2.347(\mathrm{H} \gamma)$ \\
\hline Met-65 & & & 1.946 & \\
\hline Glu-69 & & & 2.041 & \\
\hline Lys-73 & & & 2.061 & $1.855(\mathrm{H} \delta), 3.137(\mathrm{H} \epsilon)$ \\
\hline Tyr-74 & & & 1.899 & \\
\hline Ile-75 & & & & $2.032(\mathrm{Q} \delta 3)$ \\
\hline Lys-79 & & & & $0.718,0.376(\mathrm{H} \gamma), 2.291(\mathrm{H} \epsilon)$ \\
\hline Met-80 & & & 7.604 & \\
\hline Gly-84 & 8.249 & $2.651,4.309$ & & \\
\hline Lys-86 & & & 1.632 & $1.539(\mathrm{H} \delta), 2.806,2.882(\mathrm{H} \epsilon)$ \\
\hline Lys-88 & & & & $0.515(\mathrm{H} \gamma), 1.164(\mathrm{H} \delta), 1.511(\mathrm{H} \epsilon)$ \\
\hline Glu-90 & & & 1.532 & $2.026(\mathrm{H} \gamma)$ \\
\hline Arg-91 & & & 1.658 & \\
\hline Ile-95 & & & & $0.380(\mathrm{Q} \gamma)$ \\
\hline Lys-99 & & & & $0.679(\mathrm{H} \gamma), 0.102(\mathrm{H} \delta), 2.615,2.577(\mathrm{H} \epsilon)$ \\
\hline Lys-100 & & & 1.659 & $1.196(\mathrm{H} \gamma), 2.966(\mathrm{H} \epsilon)$ \\
\hline
\end{tabular}

resonances had already been assigned were predicted by calculations. After checking for the presence of the most intense among such predicted cross peaks in the experimental maps, new cross peaks were assigned and integrated and the corresponding constraints used in the following PSEUDYANA calculations. About 100 new NOE's are assigned in each step of this procedure and added to the other constraints. A total of about 800 cross peaks were assigned with this procedure.

\section{RESULTS}

\section{Sequence-Specific Assignment}

An extensive sequence-specific assignment of the protein resonances in the ${ }^{1} \mathrm{H}$ NMR spectrum of oxidized horse heart cytochrome at $293 \mathrm{~K}(0.1 \mathrm{M}$ sodium phosphate-0.15 M sodium chloride buffer, pH 5.7) was available (Feng et al., 1989). We have repeated the assignment of the protein residues under our experimental conditions $(0.1 \mathrm{M}$ phosphate buffer, $\mathrm{pH}$ 7.0). Assignments of most of the resonances were consistent with those already published (Feng et al., 1989). In total, $75 \%$ of the expected proton resonances were assigned (Supporting Information). Newly assigned resonances are given in Table 1. Of the expected signals, 68 (i.e. about $9 \%$ of the total number) are due to exchangeable protons of amino acid side chains; 14 of these were assigned, whereas the others probably could not be detected due to exchange-broadening.

\section{Secondary Structure}

Figure 1 shows the short and medium range NOEs, observed for the backbone and $\beta$ protons in the $100 \mathrm{~ms}$ NOESY map in water. Sequential $\mathrm{HN}-\mathrm{HN}$ connectivities for stretches longer than two amino acids have been observed for residues $1-4,5-14,15-25,31-33,34-38,41-43$, $47-58,60-67,72-75,87-92,93-104$. Four prolines (residues 30, 44, 71, and 76) are present in the sequence, which break the sequential $\mathrm{HN}-\mathrm{HN}$ connectivities.

Helical structures, characterized by strong $\mathrm{HN}-\mathrm{HN}$ and medium-range $\mathrm{H} \alpha-\mathrm{HN}(i, i+3), \mathrm{H} \alpha-\mathrm{H} \beta(i, i+3)$, and $\mathrm{H} \alpha-\mathrm{HN}-$ $(i, i+4)$ NOEs, are present in the segments $3-15,50-56$, $61-71,71-75,89-104$ (Figure 1). Moreover, $(i, i+3)$ and $(i, i+2)$ connectivities also have been observed for residues 14-19, thus suggesting some type of helical structure (Detlefsen et al., 1991; Marion \& Guerlesquin, 1992; Blackledge et al., 1995; Baistrocchi et al., 1996; Banci et al., 1995a).

\section{Solution Structure Calculations}

2250 NOESY and 28 1D NOE experimental constraints have been obtained. These NOESY constraints were trans- 

10
20
30
40
50
60

GDVEKGKKIFVQKCAQCXTVEKGGKHKT GPNLHGLFGRKT GQAPGFT YT DANKNKG ITWK

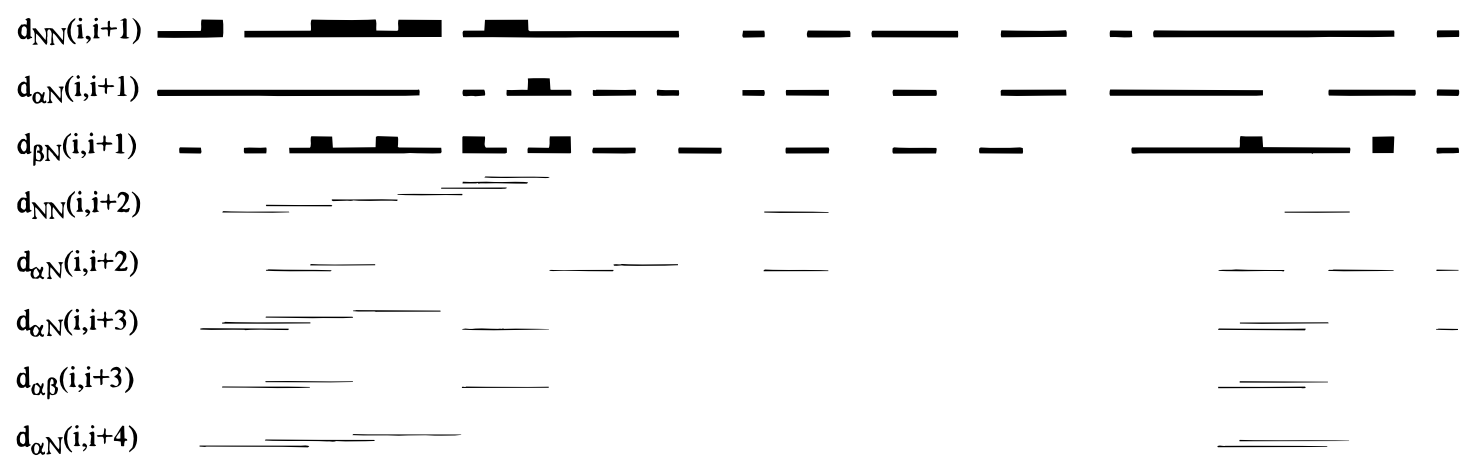

$70 \quad 80 \quad 90 \quad 100$

EETLMEYLENPKKYI PGTKMIFAGIKKKTEREDLIAYLKKATNE

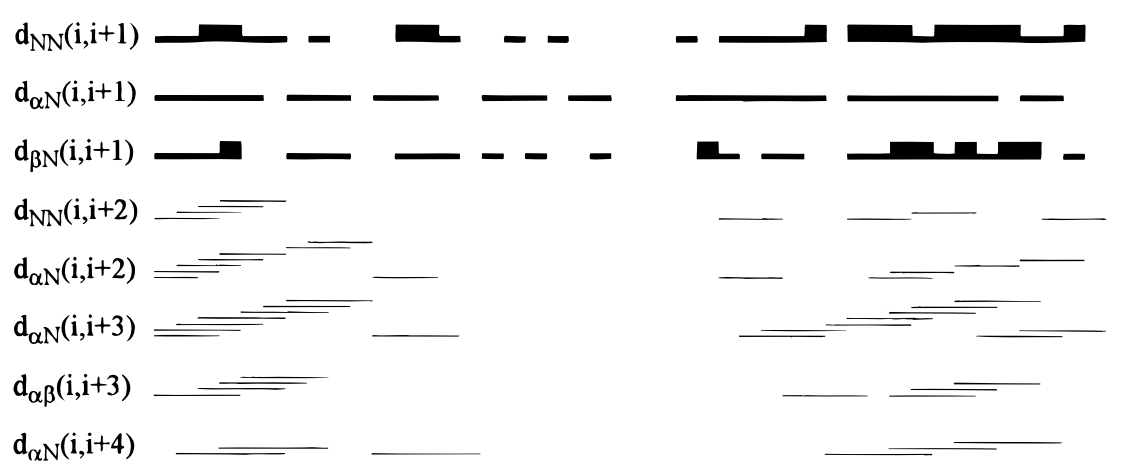

FIGURE 1: Sequential dipolar connectivities involving $\mathrm{HN}, \mathrm{H} \alpha$, and $\mathrm{H} \beta$ protons in oxidized horse heart cytochrome c. The thickness of each bar indicates relative NOE intensity.

Table 2: Summary of the Parameters Describing the Final PSEUDOREM Family of 35 Structures and Its Agreement with the Experimental Constraints

\begin{tabular}{|c|c|c|c|c|c|c|}
\hline $\begin{array}{c}\text { RMSD } \\
\text { for backbone } \\
\text { atoms }(\AA)^{a}\end{array}$ & $\begin{array}{c}\text { RMSD } \\
\text { for all heavy } \\
\text { atoms }(\AA)^{a}\end{array}$ & $\begin{array}{c}\text { largest residual } \\
\text { pseudocontact shift } \\
\text { violation }(\mathrm{ppm})\end{array}$ & $\begin{array}{l}\text { largest residual } \\
\text { NOE } \\
\text { violation }(\AA)\end{array}$ & $\begin{array}{c}\text { NOEs } \\
\text { average } \\
\text { target function }\left(\AA^{2}\right)^{b}\end{array}$ & $\begin{array}{l}\text { pseudocontact shifts } \\
\quad \text { average } \\
\text { target function }\left(\AA^{2}\right)^{b}\end{array}$ & $\begin{array}{c}\text { average } \\
\text { total energy } \\
\left.(\mathrm{kJ} \mathrm{mol})^{-1}\right)^{c}\end{array}$ \\
\hline $0.70 \pm 0.11$ & $1.21 \pm 0.14$ & 0.28 & 0.33 & $\begin{array}{c}0.97 \pm 0.11 \\
\left(130 \pm 15 \mathrm{~kJ} \mathrm{~mol}^{-1}\right)\end{array}$ & $\begin{array}{c}0.20 \pm 0.06 \\
\left(84 \pm 23 \mathrm{~kJ} \mathrm{~mol}^{-1}\right)\end{array}$ & $-3830 \pm 270$ \\
\hline
\end{tabular}

${ }^{a}$ From the mean structure. ${ }^{b}$ The penalty function obtained in PSEUDOREM calculations is given in brackets. ${ }^{c}$ Calculated with AMBER 4.0 force field.

formed into upper distance limits with the program CALIBA (Güntert et al., 1991), which uses a volume-to-distance correlation. The best calibration of observed intensities was found to be inversely proportional to the fifth or sixth power of the proton-proton distances, depending on the calibration class of NOE constraints.

1757 out of the total 2278 experimental constraints (corresponding to an average of 21.9 experimental NOEs and of 16.9 meaningful NOEs per residue) were found to be meaningful and therefore used in the PSEUDYANA calculations. The number of experimental NOE constraints per residue is shown in Figure 2A. Of the 241 pseudocontact shifts, 96 resulted to be larger than the chosen tolerance. Fourteen hydrogen bond constraints, involving slowly exchanging amide protons, consistently present in the initially calculated PSEUDYANA structures were introduced as further constraints in the last PSEUDYANA run.

The resulting family of 35 structures has RMSD values with respect to the mean structure of $0.69 \pm .12 \AA$ and 1.18 $\pm .15 \AA$ for backbone and all heavy atoms, respectively.
The total target function values for the above 35 structures are in the $1.70-2.70 \AA^{2}$ range, the contribution of the pseudocontact shift constraints being around $0.5 \AA^{2}$. The target function of NOE constraints is substantially the same also in calculations run without inclusion of shift constraints; however, the RMSD values resulting from such calculations are slightly higher. The fact that the contributions of pseudocontact shifts $\left(\approx 0.5 \AA^{2}\right)$ and NOEs $\left(1-1.5 \AA^{2}\right)$ to the target function of the final family are of the same order of magnitude indicates that it is sensible to weight the two types of constraints equally.

The family of 35 structures resulting from PSEUDOREM calculations has a RMSD with respect to the average structure of $0.70 \pm 0.11 \AA$ for backbone atoms, and of $1.21 \pm 0.14$ $\AA$ for all heavy atoms. The RMSD values per residue are reported in Figure $2 \mathrm{~B}$. The parameters quantifying the agreement between the constraints and the final family of structures are summarized in Table 2. The average total energy of the structures is also given. Figure 3 shows a schematic representation of the family. 

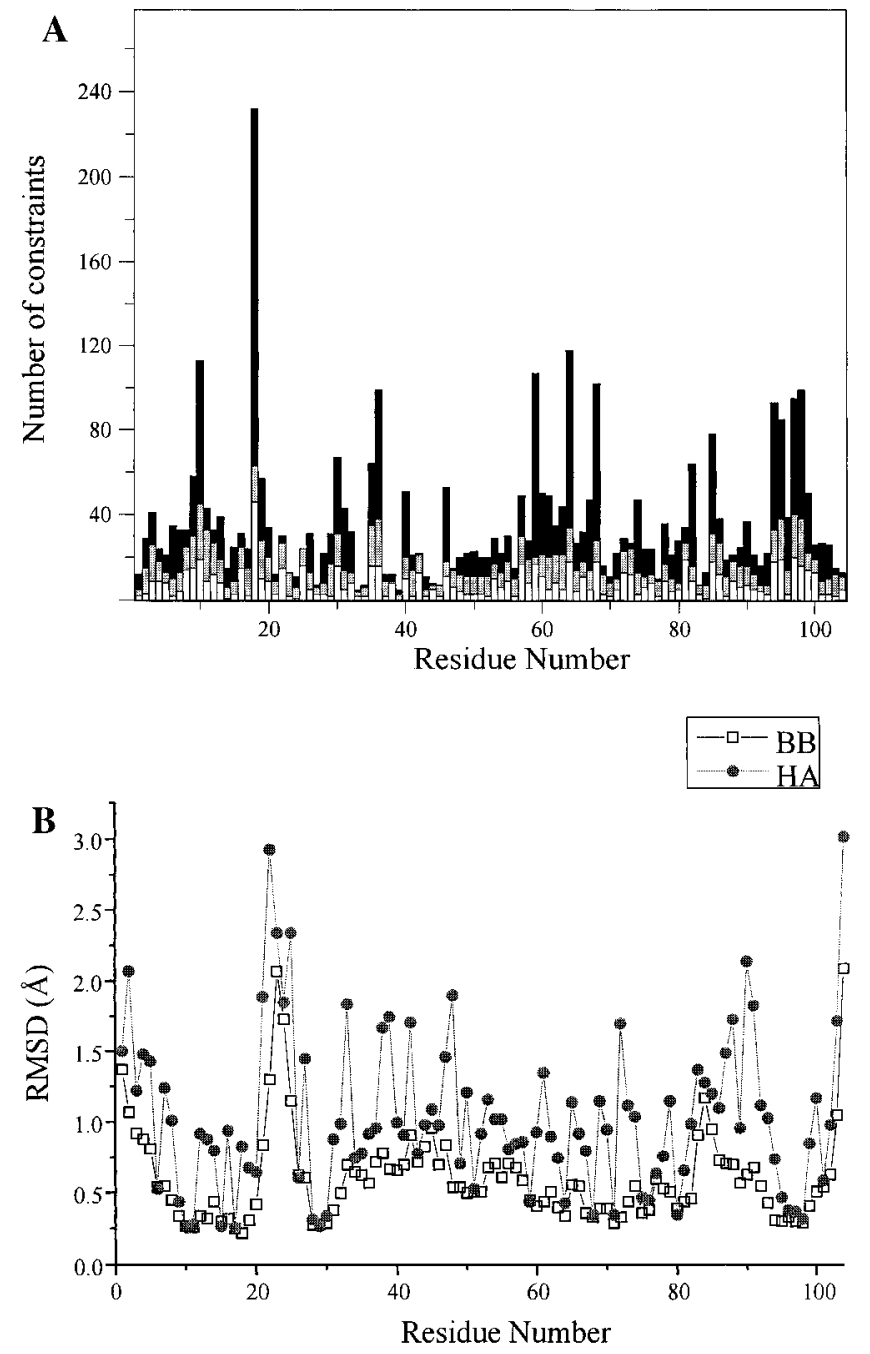

FIGURE 2: The number of observed experimental NOEs per residue (A) is compared with the RMSD values for the backbone and all heavy atoms (B). Black bars indicate long and medium range NOEs, light grey sequential NOEs, and white intraresidue NOEs.

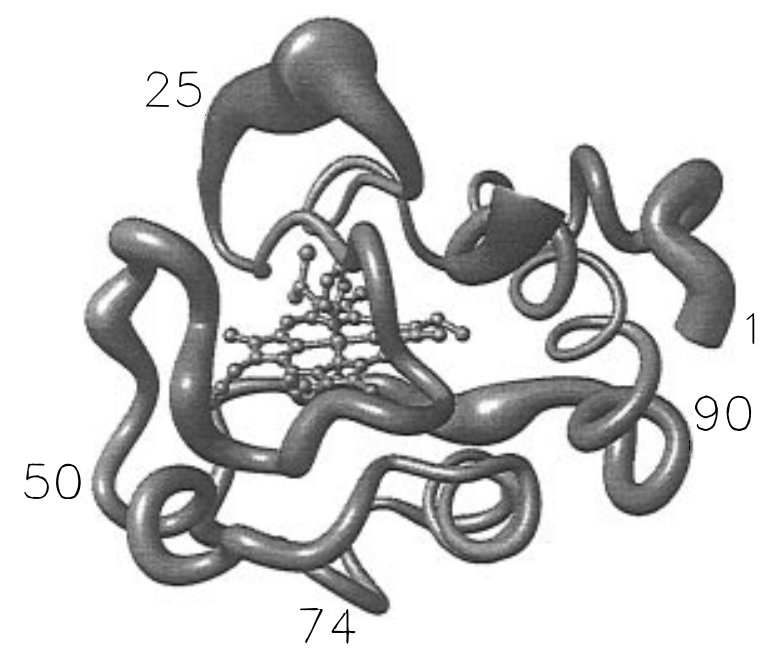

FIGURE 3: Display of the backbone of the final family of structures calculated with PSEUDOREM as a tube with variable radius. The latter is proportional to the RMSD of the backbone of each residue. The figure was generated with the program MOLMOL (Koradi et al., 1996).

\section{Magnetic Susceptibility Tensor}

The final tensor has $\Delta \chi_{\mathrm{ax}}$ and $\Delta \chi_{\mathrm{rh}}$ values of $(2.68 \pm 0.10)$ $\times 10^{-32}$ and $(1.25 \pm 0.12) \times 10^{-32} \mathrm{~m}^{3}$, respectively. The $\chi_{x x}$ and $\chi_{y y}$ axes are close, although not exactly aligned, to the directions defined by the pyrrole nitrogen atoms (the deviation is around $-10^{\circ}$ ), while the $\chi_{z z}$ axis deviates about $7^{\circ}$ from the perpendicular to the heme plane (Figure 4A). The anisotropy values are consistent with the $\mathbf{g}$ anisotropy obtained from single crystal EPR measurements (Mailer \& Taylor, 1972). The in-plane $\mathbf{g}$ tensor principal directions are, within error, the same as those of the $\chi$ tensor, whereas $\mathbf{g}_{z z}$ is reported to be, within $\pm 3^{\circ}$, orthogonal to the heme plane.

It is interesting to compare the above magnetic susceptibility parameters with those evaluated by using the same set of pseudocontact shifts and the crystal structure as input structure. The tensor parameters for the former are as follows: $\Delta \chi_{\mathrm{ax}}=2.72 \pm 0.26 \times 10^{-32} \mathrm{~m}^{3}, \Delta \chi_{\mathrm{rh}}=1.44 \pm$ $0.14 \times 10^{-32} \mathrm{~m}^{3}$, the $\chi_{z z}$ axis deviates about $18^{\circ}$ from the perpendicular to the heme plane, the $\chi_{x x}$ axis forms an angle of ca. $-12^{\circ}$ with the iron to pyrrole IV nitrogen direction (Figure 4B). It should be pointed out that the orientation of the magnetic susceptibility tensor obtained from the X-ray structure is significantly different from that obtained from the solution structure. Also noteworthy is the fact that the parameters of the tensor derived from the X-ray structure are much more sensible to small variations in the input pseudocontact shift data than the parameters obtained from the family of solution structures. Indeed, by repeating several calculations of the tensor, in which $10 \%$ of the pseudocontact shifts were randomly neglected from the input data, the $\Delta \chi_{\text {ax }}$ and $\Delta \chi_{\mathrm{rh}}$ values obtained from the X-ray structure showed variations as high as $10 \%$, whereas in the case of the tensor from the family of solution structures such variations are of the order of $2-3 \%$. The directions of the axes of the magnetic susceptibility tensor were in both cases stable within $2^{\circ}$. The above considerations suggest that the use of an incomplete set of pseudocontact shifts (e.g. because some shifts are discarded due to the possible structural differences between the reduced and oxidized species) as input for the determination of the magnetic susceptibility tensor may give rise to small but significant errors in the parameters of the latter. On the other hand the solution structure is much more stable to the incompleteness of the input set of pseudocontact shifts. This is probably due to the fact that the solution structure was calculated by using pseudocontact shifts as constraints, and thus the agreement between the structure and the shifts is higher than in the case of the X-ray structure.

For comparison purposes, it is reminded that the parameters for $S$. cerevisiae cytochrome c (obtained at $303 \mathrm{~K}$ ) are as follows: $\Delta \chi_{\mathrm{ax}}=2.22 \pm 0.07 \times 10^{-32} \mathrm{~m}^{3}, \Delta \chi_{\mathrm{rh}}=1.04 \pm$ $0.07 \times 10^{-32} \mathrm{~m}^{3}$, the $\chi_{z z}$ axis deviates about $16^{\circ}$ from the perpendicular to the heme plane, the $\chi_{x x}$ axis forms an angle of ca. $9^{\circ}$ with the iron to pyrrole IV nitrogen direction (Figure 4C) (Banci et al., 1997a).

Once the correct magnetic susceptibility tensor is available, the contact and the pseudocontact contributions to the hyperfine shifts of the heme moiety and of the iron ligands could be separated (neglecting ligand-centered dipolar shifts). The results obtained for hh cyt c and for $S$. cerevisiae cytochrome c (Banci et al., 1997a) are shown in Table 3. They are somewhat different from those already reported (Gao et al., 1990; Turner, 1993) and sensibly different from those calculated from the ${ }^{13} \mathrm{C}$ data (Turner, 1995). 

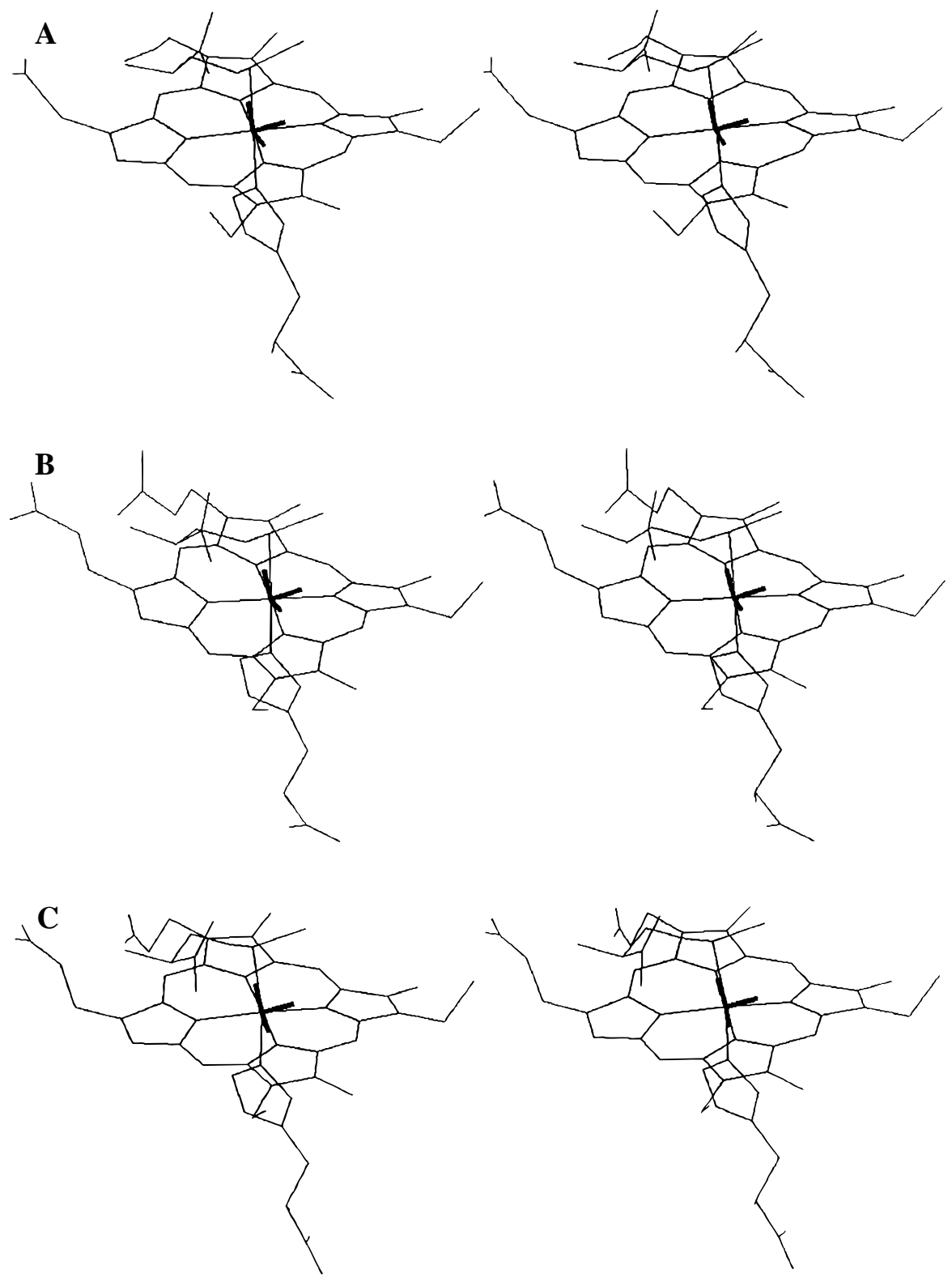

FIGURE 4: Display of the orientation with respect to the heme moiety and the axial ligands of the iron of the magnetic susceptibility tensor determined from the pseudocontact shifts used for structure calculations in this work, and using as input structure (A) the present family of solution structures (only the average structure is shown), (B) the crystal structure. The tensor obtained from the solution structure of oxidized $S$. cerevisiae iso-1-cytochrome c is shown as well (C).

The absolute values of the pseudocontact shifts of the iron ligands are somewhat larger for hh cyt c than for the $S$. cerevisiae protein. From the temperature dependence of some pseudocontact shifts, this discrepancy may at least partly be ascribed to the different experimental conditions: $293 \mathrm{~K}$ for hh cyt c versus $303 \mathrm{~K}$ for the $S$. cerevisiae protein. Indeed, it has been shown (Turner, 1993) that two electronic levels are populated at room temperature. With varying temperature, the relative populations of the two levels change, and subsequently the orientation of the axes and the $\Delta \chi$ values of the tensor vary (Turner \& Williams, 1993). The most striking difference between hh cyt c and its $S$. cerevisiae analog concerns the calculated pseudocontact shift of the $H \epsilon 1$ of the axial histidine, which is large and positive in the former, whereas it is small and negative in the latter. The different orientation of the histidine ring with respect to the heme plane (the projection of the ring in the two average structures differs of about $10^{\circ}$ ) may only account for about $20 \%$ of this discrepancy. The major contribution is due to the strong dependence of such pseudocontact shift on the deviation of the $\chi_{z z}$ axis from the normal to the heme plane (Emerson \& La Mar, 1990). Such deviation is larger in the case of the S. cerevisiae protein. In both proteins the tilt of the $\chi_{z z}$ axis is such that the $\mathrm{H} \delta 2$ proton of the axial histidine is closer to the axis itself than the $\mathrm{H} \epsilon 1$, thus justifying its positive large pseudocontact shift and its smaller dependence on the magnitude of the tilt. In both systems, the resonances of 1- $\mathrm{CH}_{3}$ and 5- $\mathrm{CH}_{3}$ show a larger pseudocontact shift than the pair $3-\mathrm{CH}_{3}$ and $8-\mathrm{CH}_{3}$. It can be noticed that the two pairs are more different in the case of hh cyt $\mathrm{c}$, as the $\chi_{y y}$ axis of the magnetic susceptibility tensor is closer to the direction $1-\mathrm{CH}_{3}$ to $5-\mathrm{CH}_{3}$.

The data of Table 3 allow us to compare the electronic structure of the heme moiety in the oxidized horse and $S$. cerevisiae cytochromes. Indeed, it appears that, with the only exception of the resonances of meso- $\beta$, meso- $\delta$, and the methyl of thioether 4 , the pattern of pseudocontact and contact shifts is rather similar in both proteins. This suggests 
Table 3: Separation of Contact and Pseudocontact Contributions to the Hyperfine Shift for hh cyt c at $293 \mathrm{~K}$ and for $S$. cerevisiae Iso-1-cytochrome $\mathrm{c}$ at $303 \mathrm{~K}$

\begin{tabular}{|c|c|c|c|c|c|c|}
\hline \multirow[b]{2}{*}{ atom name } & \multirow[b]{2}{*}{ residue name } & \multicolumn{2}{|c|}{ chemical shift } & \multirow[b]{2}{*}{$\begin{array}{l}\text { hyperfine shift } \\
(\mathrm{ppm})\end{array}$} & \multirow{2}{*}{$\begin{array}{c}\text { calculated } \\
\text { pseudocontact shift }{ }^{a} \\
(\mathrm{ppm})\end{array}$} & \multirow[b]{2}{*}{$\begin{array}{l}\text { contact shift } \\
\text { (ppm) }\end{array}$} \\
\hline & & $\begin{array}{c}\text { oxidized species } \\
(\mathrm{ppm})\end{array}$ & $\begin{array}{l}\text { reduced species } \\
(\mathrm{ppm})\end{array}$ & & & \\
\hline \multicolumn{7}{|c|}{ hh cyt $\mathrm{c}^{b}$} \\
\hline $\mathrm{H \alpha}$ & Met & 2.77 & 3.09 & -0.32 & $-0.03 \pm 0.30$ & -0.29 \\
\hline$\epsilon-\mathrm{CH}_{3}$ & Met & -24.7 & -3.30 & -21.4 & $4.42 \pm 1.4$ & -25.8 \\
\hline $\mathrm{H} \alpha$ & $\mathrm{His}$ & 9.10 & 3.50 & 5.30 & $4.28 \pm 0.04$ & 1.04 \\
\hline $\mathrm{H} \delta 2$ & His & 24.6 & 0.14 & 24.5 & $25.7 \pm 0.45$ & -1.20 \\
\hline $\mathrm{H} \in 1$ & His & -25.7 & 0.51 & -26.2 & $12.9 \pm 0.64$ & -39.0 \\
\hline $8-\mathrm{CH}_{3}$ & heme & 35.7 & 2.16 & 33.5 & $-1.86 \pm 0.04$ & 35.4 \\
\hline meso- $\delta \mathrm{H}$ & heme & 2.11 & 9.06 & -6.95 & $-9.06 \pm 0.15$ & 2.11 \\
\hline $1-\mathrm{CH}_{3}$ & heme & 6.81 & 3.46 & 3.35 & $-4.38 \pm 0.07$ & 7.73 \\
\hline $2-\mathrm{H \alpha}$ & heme & -1.33 & 5.20 & -6.53 & $-4.73 \pm 0.05$ & -1.8 \\
\hline $2-\mathrm{CH}_{3}$ & heme & -2.63 & 1.46 & -4.09 & $-3.09 \pm 0.02$ & -1.0 \\
\hline meso- $\alpha \mathrm{H}$ & heme & 1.40 & 9.30 & -7.90 & $-6.31 \pm 0.09$ & -1.6 \\
\hline $3-\mathrm{CH}_{3}$ & heme & 32.8 & 3.84 & 29.0 & $-1.15 \pm 0.01$ & 30.1 \\
\hline $4-\mathrm{H \alpha}$ & heme & 2.09 & 6.30 & -4.21 & $-2.70 \pm 0.09$ & -1.51 \\
\hline $4-\mathrm{CH}_{3}$ & heme & 3.05 & 2.57 & 0.48 & $-1.37 \pm 0.02$ & 1.85 \\
\hline meso- $\beta \mathrm{H}$ & heme & -0.92 & 9.61 & -10.5 & $-10.63 \pm 0.13$ & 0.13 \\
\hline $5-\mathrm{CH}_{3}$ & heme & 9.72 & 3.58 & 6.14 & $-4.86 \pm 0.03$ & 11.0 \\
\hline meso- $\gamma \mathrm{H}$ & heme & 7.50 & 9.64 & -2.14 & $-6.25 \pm 0.09$ & 4.11 \\
\hline \multicolumn{7}{|c|}{ S. cerevisiae cytochrome $\mathrm{c}^{c}$} \\
\hline $\mathrm{H} \alpha$ & Met & - & 3.12 & - & $0.97 \pm 0.63$ & - \\
\hline$\epsilon-\mathrm{CH}_{3}$ & Met & -22.4 & -3.18 & -19.2 & $3.43 \pm 1.7$ & -22.6 \\
\hline $\mathrm{H} \alpha$ & $\mathrm{His}$ & 8.82 & 3.60 & 5.22 & $3.93 \pm 0.09$ & 1.29 \\
\hline $\mathrm{H} \delta 2$ & His & 24.0 & 0.093 & 23.9 & $21.5 \pm 0.43$ & 2.40 \\
\hline $\mathrm{H} \epsilon 1$ & His & -25.1 & 0.51 & -25.6 & $-0.79 \pm 0.43$ & -24.8 \\
\hline $8-\mathrm{CH}_{3}$ & heme & 34.7 & 2.29 & 32.4 & $-0.96 \pm 0.02$ & 33.4 \\
\hline$m e s o-\delta \mathrm{H}$ & heme & 2.34 & 9.18 & -6.84 & $-5.36 \pm 0.11$ & -1.48 \\
\hline $1-\mathrm{CH}_{3}$ & heme & 8.01 & 3.49 & 4.52 & $-2.94 \pm 0.04$ & 7.46 \\
\hline $2-\mathrm{H \alpha}$ & heme & -0.70 & 5.23 & -5.93 & $-4.28 \pm 0.08$ & -1.65 \\
\hline $2-\mathrm{CH}_{3}$ & heme & -2.10 & 1.50 & -3.60 & $-3.17 \pm 0.06$ & -0.43 \\
\hline meso- $\alpha \mathrm{H}$ & heme & 2.66 & 9.35 & -6.69 & $-6.77 \pm 0.08$ & -0.08 \\
\hline $3-\mathrm{CH}_{3}$ & heme & 31.0 & 3.88 & 27.1 & $-1.18 \pm 0.04$ & 28.3 \\
\hline $4-\mathrm{H \alpha}$ & heme & 2.86 & 6.39 & -3.53 & $-1.53 \pm 0.02$ & -2.00 \\
\hline $4-\mathrm{CH}_{3}$ & heme & 2.08 & 2.55 & -0.47 & $-0.46 \pm 0.01$ & -0.01 \\
\hline meso- $\beta \mathrm{H}$ & heme & 1.71 & 9.72 & -8.01 & $-5.58 \pm 0.03$ & -2.43 \\
\hline $5-\mathrm{CH}_{3}$ & heme & 11.0 & 3.54 & 7.46 & $-3.54 \pm 0.02$ & 11.0 \\
\hline meso- $\gamma \mathrm{H}$ & heme & 8.03 & 9.64 & -1.61 & $-5.88 \pm 0.10$ & 4.27 \\
\hline
\end{tabular}

${ }^{a}$ The calculated pseudocontact shift is the average pseudocontact shift calculated over each member of the family of structures constituting the solution structure. The standard deviation of the calculated pseudocontact shift is reported. The tensor parameters for $S$. cerevisiae cytochrome c have been taken from Banci et al. (1997a). ${ }^{b}$ The shifts of the reduced species are taken from Santos and Turner (1992). ${ }^{c}$ The shifts of the oxidized species are taken from Gao et al. (1990) and Banci et al. (1997a). The shifts of the reduced species are taken from Gao et al (1990).

that the electronic structure of the heme moiety in the two oxidized proteins is similar. It is also interesting to notice the large upfield contact shift of the methyl group of the axial methionine. This is at variance with the case of cysteine $\beta \mathrm{CH}_{2}$ protons in iron-sulfur systems, which are downfield shifted (Bertini et al., 1995). In the latter systems, where the iron(III) and iron(II) are high spin, positive electron spin density is delocalized directly from the iron orbitals onto the sulfur orbitals of the cysteine and transferred to the proton $1 \mathrm{~s}$ orbital. In the case of low spin iron(III), such as the present case, the $\sigma$ bond between the iron and the sulfur results from the interaction of the empty $d_{z}{ }^{2}$ orbital of the iron with an electron pair of the sulfur. Electron spin density is transferred from the half-filled orbital of the iron to the orbitals of the sulfur through spin polarization of the electrons in the $\sigma$ bonding orbital. The sign of the transferred electron spin density is thus reversed with respect to that in ironsulfur systems, and so is the sign of the contact shift. Finally, it is to be noted the contact contribution to the chemical shift for the $\mathrm{H} \alpha$ protons of the axial ligands in both the $S$. cerevisiae and the present cytochrome is larger than the tolerance used in calculations. This provides an a posteriori justification for having neglected all pseudocontact shifts relative to such residues.

\section{Structure Analysis}

RMSD values deal with the precision of the calculated structure and should be discussed with respect to the number of experimental constraints. The present solution structure of oxidized horse cyt c, obtained without isotope labeling, has a relatively high resolution also thanks to the use of the pseudocontact shifts together with NOEs constraints. The value of $0.70 \AA$ for the backbone RMSD is the result of large regions with very high definition (RMSD values $<0.5$ A) together with some less defined regions, which correspond to those with a lower number of NOEs (Figure 2). The regions with highest disorder involve residues $21-26,44-$ 47, 83-86. The first fragment corresponds to an external loop, whose residues are intrinsically poor in NOEs; a loop with the same features is also present in $S$. cerevisiae cytochrome c. Indeed, if residues $21-26$ are excluded from RMSD calculations, the value for backbone atoms drops to $0.60 \AA$. The relatively high RMSD values for the backbone of residues 44-47 may be due to the fact that these residues 
also belong to a loop pointing toward the exterior of the protein.

The quality of the present family of structures has been also analyzed in terms of ideal geometry parameters with the PROCHECK-NMR program (Laskowski et al., 1996). The quality of the structures was checked through the Ramachandran plot. Among the 87 residues per structure which are "meaningful" for the Ramachandran plot (i.e., nonglycine, non-proline, and nonterminal residues), $56.3 \%$ fall in the most favored regions, $35.6 \%$ in the additional allowed regions, and $6.9 \%$ fall in generously allowed regions. Only one residue (Glu42) falls in a disallowed region. Glu42 is a residue at the surface of the protein, far from the heme cleft.

The elements of secondary structure are similar to those of the X-ray structure of the same protein (Figure 5). Indeed, in the present solution structure $\alpha$-helices are observed at residues $2-7,9-14,49-54,60-69,89-102$ and a $3_{10}$ helix is observed at residues 70-74. Analogously, helices are reported for residues $2-14,49-55,60-68,70-75,87-$ 104 in the X-ray structure of oxidized hh cyt. The different length for the first helix can be explained with the fact that in the present solution structure the backbone in the $7-8$ range has a distorted helical conformation, which does not meet the requirements to be classified as a helix by the Procheck program. We believe that this is mainly due to the lack of few NOEs more than to an actual difference between the solution and the crystal. Another difference for the backbone in the two structures is observed for the residues between Pro71 and Pro76: in the X-ray structure these four residues form a regular, although quite short, $\alpha$-helix, whereas in solution they have a 310 helical conformation.

The assessment of the quality of a solution structure can also be obtained by the evaluation of the number of assigned connectivities with respect to the expected ones (for a given set of assigned protons). This should give an idea about the accuracy of the calculated structure. We have analyzed this aspect by comparing the NOESY cross peaks calculated by the program CORMA (see Methods) using the energyminimized average structure. Paramagnetic effects were neglected. In particular we checked that all the cross peaks used in structural calculations corresponded to an expected peak. Moreover, we checked that all the expected cross peaks (above a certain intensity threshold) have been identified in our spectrum. But, most importantly, we verified that all the well resolved peaks above a reasonable contour level in the 2D NOESY maps have been assigned, integrated, and used in the structure calculations.

\section{DISCUSSION}

Structural differences between the two redox forms of an electron transfer protein are relevant for the understanding of the electron transfer pathway and energetics. Several spectroscopic studies, especially NMR investigations, have pointed out differences in the exchange behavior of amide protons (Schejter, 1996). The exchange data under different experimental conditions indicate increased proton lability in the oxidized form for residues around the heme axial ligands and have been discussed with respect to the unfolding mechanism (Marmorino et al., 1993; Bai et al., 1995; Banci et al., 1997a).
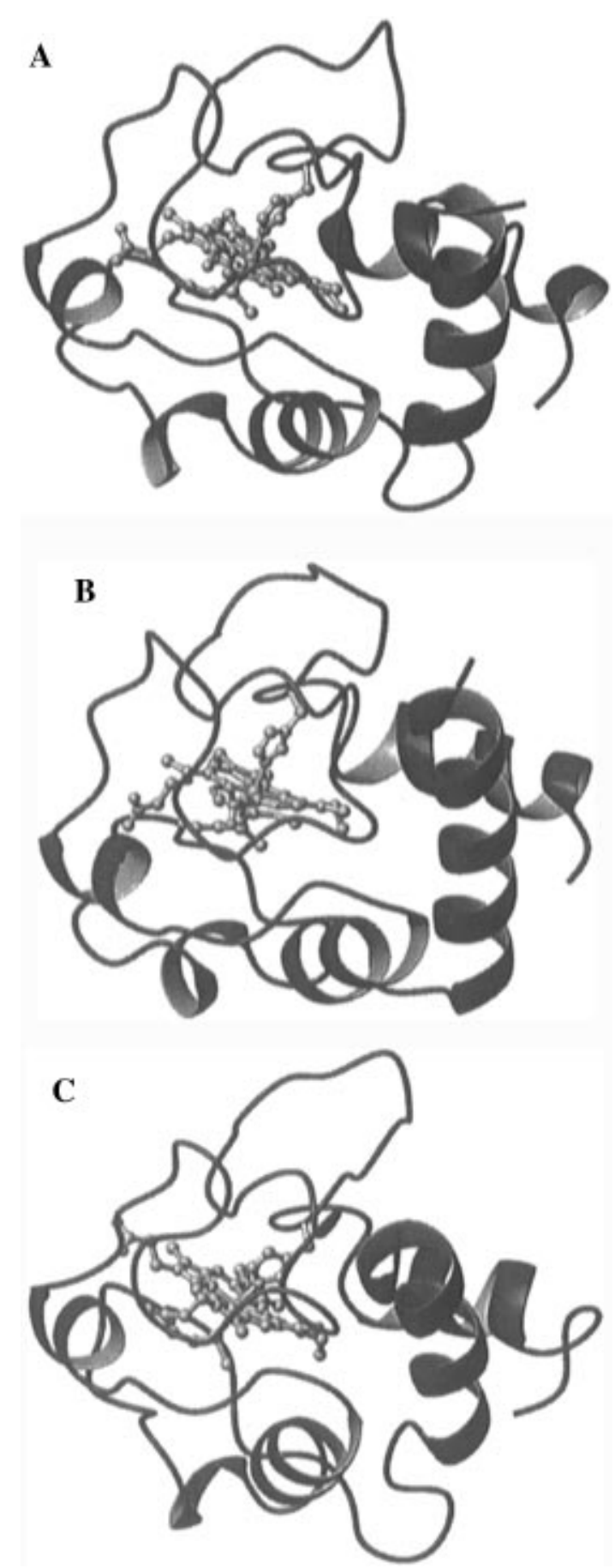

FIGURE 5: Comparison of the energy-minimized average solution structure (A) with the crystallographic structure (B) of oxidized horse heart cytochrome $\mathrm{c}$. The solution structure of the reduced form is also reported (C). The figures were generated with the program MOLMOL (Koradi et al., 1996).

Beside the above considerations, the first structural information comes from X-ray crystallography. High resolution crystal structures of five cytochromes c have been solved (Louie \& Brayer, 1990; Berghuis \& Brayer, 1992; Bushnell et al., 1990; Takano \& Dickerson, 1981b; Takano \& Dickerson, 1981a; Murphy et al., 1992; Ochi et al., 1983). In the case of tuna and $S$. cerevisiae cytochromes c the structures have been determined for both redox states (Takano \& Dickerson, 1981b; Takano \& Dickerson, 1981a; Louie \& Brayer, 1990; Berghuis \& Brayer, 1992). The major differences observed by comparing the proteins in the two redox states involve the heme distortion, the orientation of 
the axial histidine ligand, formation and breaking of a few hydrogen bonds involving heme propionate-7, and variations in some water molecules surrounding the heme. Most of these features are related to subtle changes, and the question arises whether they really reflect actual differences between the two redox forms in solution or they are induced by the crystal formation. NMR spectroscopy was first used to infer structural variations upon oxidation from the analysis of pseudocontact shifts using the crystal structure of the protein as the actual structure in solution (Gao et al., 1991). Pseudocontact shifts have been taken as the difference between the experimental shift in the oxidized cytochrome and that of the same proton in the reduced form. Deviations of about $0.6-1.5 \mathrm{ppm}$ of the calculated pseudocontact shifts with respect to the observed ones were taken as an evidence of a structural difference between the reduced and oxidized forms which makes the diamagnetic reference not correct for the evaluation of the pseudocontact shifts. Most recently, it has been shown that discrepancies can be most probably related to the incorrectness of using the crystal structure as the actual structure (Banci et al., 1996).

The comparison of the solution structures of oxidized and reduced $S$. cerevisiae cytochrome c pointed out a substantial similarity between them (Banci et al., 1997a). Moreover, both structures are, within the indetermination of the family, substantially similar to the corresponding X-ray structures (Baistrocchi et al., 1996; Banci et al., 1997a). The similarity is especially evident for the backbone conformation. Within the above frame the recently published data of Qi et al. (Qi et al., 1996) appear quite unexpected and suggest a different behavior for the horse heart cytochrome $\mathrm{c}$ with respect to the $S$. cerevisiae protein. The present results, at variance, show a much higher similarity between the solution and crystal structures of hh cyt c (Bushnell et al., 1990) and with the solution structure of reduced hh cyt c (Qi et al., 1994) (Figure 5). In the absence of the coordinates and of the experimental structural constraints, we may ascribe the differences between the present structure and that previously reported (Qi et al., 1996) as simply due to the better structure refinement of the present one. Indeed, after running PROCHECK (Laskowski et al., 1993) on the solution structure of reduced hh cyt c (Qi et al., 1994) deposited in the Protein Data Bank, very similar secondary structure elements are observed for the two solution structures. The $5-14,49-55,60-68,91-103 \alpha$-helices and a 70-74 $310^{-}$ helix are observed in the reduced protein, which compare to the $\alpha$-helices $2-7,9-14,49-54,60-69,89-102$ and the $3_{10}$ helix $70-74$ of the present structure. As pointed out in Results, the two helices $2-7$ and $9-14$ belong to a single helical structure, which is slightly distorted in the region $7-8$. The fact that in the reduced hh cyt $\mathrm{c}$ the $\mathrm{C}$-term helix is shorter (i.e. it involves residues 91-103 instead of 88-103) may be due to the lack of a few NOEs at the beginning of this helix. A major difference in the backbone conformation for the two forms of hh cyt $\mathrm{c}$ is observed for the loop 3238 (Figure 5). The conformation of the above loop is the same in the present structure and in the crystal structure. Finally, a small rotation of the plane of His 18 of $17 \pm 8^{\circ}$ is observed between our structure and that of the reduced protein, the conformation of the backbone being unchanged. Figure 6 shows a close-up of the two axial ligands of the iron for the final PSEUDOREM family of 35 structures.

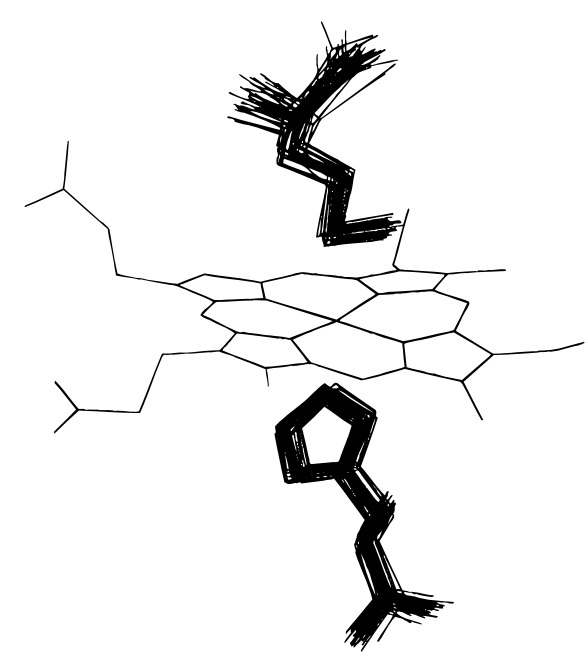

FIGURE 6: Backbone and side chain heavy atoms of the two axial ligands of the iron for the PSEUDOREM family. Only the average heme plane is shown.

As discussed in the Results section, the elements of secondary structure observed in the X-ray structure are all maintained in the present solution structure. Some small differences in the backbone conformation are noticed for a few residues (e.g. Pro76, Lys86). Regarding the two axial ligands, the conformation of the backbone and the side-chain of Met80 is the same in the crystal and in the solution structure, whereas a rotation of the plane of His 18 of $17 \pm$ $8^{\circ}$ is observed. The latter change is well documented by 13 NOEs connecting the ring protons to surrounding groups (Figure 6).

In the light of the results found in $S$. cerevisiae cytochrome c, we have also analyzed the conformation of propionate- 7 and surrounding residues in horse cyt $\mathrm{c}$. The conformation of the propionate is different in the two oxidation states: in the reduced form the chain is pointing toward the distal site, whereas in the oxidized form it is pointing toward the proximal site. Furthermore, in the reduced protein in solution propionate-7 interacts with Asn52, as it results from the coordinates deposited at the Protein Data Bank. In the oxidized form in solution, the carboxylate is disordered, although the $\alpha$ and $\beta$ protons of the propionate are well defined by a total of 40 NOE's. In the X-ray structure of the oxidized form, propionate-7 is well ordered and H-bonded to the NH proton of the ring of Trp59, the $\mathrm{H} \delta 2$ of Asn52, and the amide proton of Gly41.

In summary, the present results show that all elements of secondary structure are maintained both when passing from the crystal to the solution, and from the reduced to the oxidized state. The structural changes occurring upon oxidation or reduction of the protein is limited to the rearrangement of the loop involving residues $32-38$ and of the average position of propionate- 7 and to a readjustment of the hydrogen bond network of the latter. A difference in the average position and hydrogen bond network of propionate-7 in solution occurring upon change of the oxidation state of the heme moiety has been observed in other cytochrome c's (Banci et al., 1997a; Banci et al., 1997b). However, such rearrangement is not the same for cytochromes of different origin.

The simultaneous use of pseudocontact shifts and NOE constraints yielded a well defined structure. The two sets of constraints are found to be essentially consistent with each 
other. Finally, the procedure described in this work allowed us to obtain a reliable magnetic susceptibility tensor, which is fully consistent with the structure.

\section{ACKNOWLEDGMENT}

We thank Peter Güntert for the helpful discussions on the DYANA program.

\section{SUPPORTING INFORMATION AVAILABLE}

Tables containing proton assignments (shift values at 293 $\mathrm{K})$, experimental NOE intensities, hydrogen bond, 1D NOE, and pseudocontact shift constraints used for the structure calculations (26 pages). Ordering information is given on any current masthead page.

\section{REFERENCES}

Bai, Y. W., Sosnick, T. R., Mayne, L., \& Englander, S. W. (1995) Science 269, 192-197.

Baistrocchi, P., Banci, L., Bertini, I., Turano, P., Bren, K. L., \& Gray, H. B. (1996) Biochemistry 35, 13788-13796.

Banci, L., Bertini, I., Luchinat, C., Piccioli, M., Scozzafava, A., \& Turano, P. (1989) Inorg. Chem. 28, 4650-4656.

Banci, L., Bertini, I., Bren, K. L., Gray, H. B., Sompornpisut, P., \& Turano, P. (1995a) Biochemistry 34, 11385-11398.

Banci, L., Bertini, I., Pierattelli, R., Tien, M., \& Vila, A. J. (1995b) J. Am. Chem. Soc. 117, 8659-8667.

Banci, L., Bertini, I., Bren, K. L., Cremonini, M. A., Gray, H. B., Luchinat, C., \& Turano, P. (1996) J. Biol. Inorg. Chem. 1, 117126.

Banci, L., Bertini, I., Bren, K. L., Gray, H. B., Sompornpisut, P., \& Turano, P. (1997a) Biochemistry (in press).

Banci, L., Bertini, I., De la Rosa, M. A., Koulougliotis, D., Navarro, J. A., \& Walter, O. (1997b) The solution structure of oxidized cytochrome $c_{6}$ from the green alga Monoraphidium braunii (unpublished).

Banci, L., Bertini, I., Gori Savellini, G., Romagnoli, A., Turano, P., Cremonini, M. A., Luchinat, C., \& Gray, H. B. (1997c) Proteins: Structure, Function, and Genetics (in press).

Bax, A., \& Davis, D. G. (1985) J. Magn. Reson. 65, 355-360.

Berghuis, A. M., \& Brayer, G. D. (1992) J. Mol. Biol. 223, 959976.

Bertini, I., Ciurli, S., \& Luchinat, C. (1995) in Structure and Bonding, pp 1-54, Vol. 83, Springer-Verlag, Berlin, Heidelberg.

Bertini, I., \& Luchinat, C. (1986) NMR of paramagnetic molecules in biological systems, Benjamin/Cummings, Menlo Park, CA.

Bertini, I., \& Luchinat, C. (1996) NMR of paramagnetic substances, Coordination Chemistry Reviews 150, Elsevier, Amsterdam.

Bertini, I., \& Turano, P. (1994) in NMR of paramagnetic macromolecules. NATO ASI Series (La Mar, G. N., Ed.) Kluwer Academic, Dordrecht.

Blackledge, M. J., Medvedeva, S., Poncin, M., Guerlesquin, F., Bruschi, M., \& Marion, D. (1995) J. Mol. Biol. 245, 661-681.

Borgias, B., Thomas, P. D., \& James, T. L. (1989) Complete Relaxation Matrix Analysis (CORMA), University of California, San Francisco, CA.

Bushnell, G. W., Louie, G. V., \& Brayer, G. D. (1990) J. Mol. Biol. 214, 585-595.

Detlefsen, D. J., Thanabal, V., Pecoraro, V. L., \& Wagner, G. (1991) Biochemistry 30, 9040-9046.

Eccles, C., Güntert, P., Billeter, M., \& Wüthrich, K. (1991) J. Biomol. NMR 1, 111-130.

Emerson, S. D., \& La Mar, G. N. (1990) Biochemistry 29, 15561566.

Feng, Y. Q., Roder, H., Englander, S. W., Wand, A. J., \& Di Stefano, D. L. (1989) Biochemistry 28, 195-203.

Feng, Y. Q., Roder, H., \& Englander, S. W. (1990) Biochemistry 29, 3494-3504.
Gao, Y., Boyd, J., Williams, R. J. P., \& Pielak, G. J. (1990) Biochemistry 29, 6994-7003.

Gao, Y., Boyd, J., Pielak, G. J., \& Williams, R. J. P. (1991) Biochemistry 30, 1928-1934.

Gochin, M., \& Roder, H. (1995) Protein Sci. 4, 296-305.

Güntert, P., Braun, W., \& Wüthrich, K. (1991) J. Mol. Biol. 217, 517-530.

Güntert, P., Mumenthaler, C., \& Wüthrich, K. (1997) Torsion Angle Dynamics for NMR Structure Calculation with the new program DYANA (unpublished).

Güntert, P., \& Wüthrich, K. (1991) J. Biomol. NMR 1, 447-456.

Inubushi, T., \& Becker, E. D. (1983) J. Magn. Reson. 51, 128133.

Koradi, R., Billeter, M., \& Wüthrich, K. (1996) J. Mol. Graphics $14,51-55$.

La Mar, G. N., Horrocks, W. D. W., \& Holm, R. H., Eds. (1973) NMR of Paramagnetic Molecules, Academic Press, New York.

La Mar, G. N., Chen, Z. G., Vyas, K., \& McPherson, A. D. (1995) J. Am. Chem. Soc. 117, 411-419.

Langen, R., Brayer, G. D., Berghuis, A. M., McLendon, G., Sherman, F., \& Warshel, A. (1992) J. Mol. Biol. 224, 589-600.

Laskowski, R. A., MacArthur, M. W., Moss, D. S., \& Thornton, J. M. (1993) J. Appl. Crystallogr. 26, 283-291.

Laskowski, R. A., Rullmann, J. A. C., MacArthur, M. W., Kaptein, R., \& Thornton, J. M. (1996) J. Biomol. NMR 8, 477-486.

Louie, G. V., \& Brayer, G. D. (1990) J. Mol. Biol. 214, 527-555.

Macura, S., Wüthrich, K., \& Ernst, R. R. (1982) J. Magn. Reson. 47, 351-357.

Mailer, C., \& Taylor, C. P. S. (1972) Can. J. Biochem. 85, 10481055.

Marion, D., \& Guerlesquin, F. (1992) Biochemistry 31, 8171-8179.

Marion, D., \& Wüthrich, K. (1983) Biochem. Biophys. Res. Commun. 113, 967-974.

Marmorino, J. L., Auld, D. S., Betz, D. S., Doyle, D. F., Young, G. B., \& Pielak, G. J. (1993) Protein Sci. 2, 1966-1974.

Murphy, M. E. P., Nall, B. T., \& Brayer, G. D. (1992) J. Mol. Biol. 227, 160-176.

Ochi, H., Hata, Y., Tanaka, N., Kakudo, M., Sakuri, T., Achara, S., \& Morita, Y. (1983) J. Mol. Biol. 166, 407-418.

Pearlman, D. A., \& Case, D. A. (1991) SANDER, University of California, San Francisco, CA.

Pelletier, H., \& Kraut, J. (1992) Science 258, 1748-1755.

Qi, P. X., Di Stefano, D. L., \& Wand, A. J. (1994) Biochemistry 33, 6408-6417.

Qi, P. X., Beckman, R. A., \& Wand, A. J. (1996) Biochemistry 35, 12275-12286.

Rajarathnam, K., La Mar, G. N., Chiu, M. L., \& Sligar, S. G. (1992) J. Am. Chem. Soc. 114, 9048-9058.

Santos, H., \& Turner, D. L. (1992) Eur. J. Biochem. 206, 721728.

Schejter, A. (1996) in Oxidation State-Dependent Properties of cytochrome c (Scott, R. A., \& Mauk, A. G, Eds.) pp 335-345, University Science Books, Sausalito, CA.

Scott, R. A., \& Mauk, A. G., Eds. (1996) Cytochrome c. A multidisciplinary approach, University Science Books, Sausalito, CA.

Takano, T., \& Dickerson, R. E. (1981a) J. Mol. Biol. 153, 95155.

Takano, T., \& Dickerson, R. E. (1981b) J. Mol. Biol. 153, 79-94.

Turner, D. L. (1993) Eur. J. Biochem. 211, 563-568.

Turner, D. L. (1995) Eur. J. Biochem. 227, 829-837.

Turner, D. L., \& Williams, R. J. P. (1993) Eur. J. Biochem. 211, $555-562$.

Wand, A. J., Di Stefano, D. L., Feng, Y. Q., Roder, H., \& Englander, S. W. (1989) Biochemistry 28, 186-194.

Williams, G., Clayden, N. J., Moore, G. R., \& Williams, R. J. P. (1985) J. Mol. Biol. 183, 447-460.

BI970724W 